\title{
Inflation in (Super-)renormalizable Gravity
}

\author{
Fabio Briscese, ${ }^{1,2}$ Antonino Marcianò ${ }^{3}$ Leonardo Modesto, ${ }^{4}$ and Emmanuel N. Saridakis ${ }^{5,6}$ \\ ${ }^{1}$ Istituto Nazionale di Alta Matematica Francesco Severi, \\ Gruppo Nazionale di Fisica Matematica, Città Universitaria, P.le A. Moro 5, 00185 Rome, EU \\ ${ }^{2}$ SBAI, Sezione di Matematica, Sapienza Università di Roma, Via Antonio Scarpa 16, 00161 Rome, EU \\ ${ }^{3}$ Department of Physics \& Astronomy, HB 6127 Wilder Lab, Dartmouth College, Hanover, NH 03755, USA \\ ${ }^{4}$ Department of Physics 85 Center for Field Theory and Particle Physics, Fudan University, 200433 Shanghai, China \\ ${ }^{5}$ Physics Division, National Technical University of Athens, 15780 Zografou Campus, Athens, EU \\ ${ }^{6}$ Instituto de Física, Pontificia Universidad Católica de Valparaíso, Casilla 4950, Valparaíso, Chile
}

\begin{abstract}
We investigate a (super-)renormalizable and ghost-free theory of gravity, showing that under a natural (exponential) ansatz of the form factor and a suitable truncation it can give rise to the Starobinsky inflationary theory in cosmological frameworks, and thus offering a theoretical justification of its origin. We study the corresponding inflationary evolution and we examine the generation of curvature perturbations, adapting the $f(R)$-like equations in a symmetry-reduced FLRW metric. Furthermore, we analyze how the ultraviolet regime of a simply renormalizable and unitary theory of gravity is also compatible with the Starobinsky action, and hence we show that such a theory could account for an inflationary phase of the Universe in the ultraviolet regime.
\end{abstract}

\section{INTRODUCTION}

Recently a new gravitational action principle has been introduced and/or reconsidered in order to alleviate the shortcomings of Einstein theory [1 -5$]$. This theory, and the corresponding approach to quantum gravity partly inspired by the Cornish and Moffat papers [6], is constructed in order to fulfill a synthesis of minimal hypotheses: (i) classical solutions must be singularity-free; (ii) Einstein-Hilbert action should be a good approximation of the theory at a much smaller energy scale than the Planck mass; (iii) the spacetime dimension has to decrease with the energy in order to have a complete quantum gravity theory in the ultraviolet regime; (iv) the theory has to be perturbatively renormalizable at the quantum level (this requirement is strongly related to the previous one), (v) the theory has to be unitary, with no other pole in the propagator in addition to the graviton, (vi) spacetime is a single continuum of space and time and in particular the Lorentz invariance is not broken, consistently with observations. This (super-)renormalizable gravitational theory is therefore consistent with the basic requirements of quantum gravity.

On the other hand, a large amount of research has been dedicated to the construction of modified gravitational theories, which are capable of describing the observed late-time acceleration and the detected signatures of inflation (see [7] and references therein). Amongst them the $f(R)$-gravity (see [8] and references therein) is perhaps the most investigated one, with interesting implications in spite of its simple form. However, although the above modified-gravity scenarios exhibit interesting phenomenology in agreement with observations, their construction is mainly artificial, without a theoretical justification, hoping that an underlying fundamental theory of quantum gravity, unknown up to now, would eventually provide them as low-energy limits. In the same lines, gravitational modifications based on the inclusion of finite higher derivatives (see [9] and references therein) can also have interesting cosmological implications. For instance, non local theories with lagrangian density $\mathcal{L}=F\left(R, \square R, \square^{2} R, \ldots, \square^{m} R, \square^{-} R, \ldots, \square^{-m} R\right)$ have a classical equivalent scalar representation obtained introducing $2(m+n)$ auxiliary scalar fields, which is capable to explain inflation and dark energy in a unified framework [10, 11], see also [12] for a complete review. However, all these extensions of general relativity seems to be artificial too, with the main exception being the Horava-Lifshitz gravity, in which the higher-order derivatives are added following the fundamental symmetries of the theory [13] and the inclusion of fermionic matter has been conjectured to be responsible for the perturbatively consistent UV behavior [14]. See also [15] for an investigation of non-locally modified models of gravity, compatible with quantum loop corrections, in light of theory application as a mechanism for current cosmic acceleration.

In the present work we are interested in providing a theoretical justification of some of $f(R)$-gravitational scenarios, in the context of the above (super-)renormalizable gravitational theory. In particular, we show that this theory, which in principle contains infinite number of higher derivative terms, under a suitable truncation can give rise to the famous Starobinsky theory [16]

$$
\mathcal{L}=R+\epsilon R^{2},
$$

which proves to be a viable inflationary model in perfect agreement with current observations. Especially the recently released WMAP nine-year results suggest that Starobinsky model is the one that best describes the data, comparing to scalar field inflation [17]. Starobinsky model was first introduced due to one loop contributions of conformally covariant matter fields to the Einsteinian theory of gravity [16], but it was subsequently shown that it can be derived in the framework of superstring theory [18 20], or that it can be embedded in M-theory [21] and in $F(R)$ supergravity [22]. In the following we show that the Starobinsky model can alternatively and naturally 
emerge from a consistent truncation of the aforementioned (super-)renormalizable quantum gravity models, when a proper form-factor is assumed.

The plan of the work is as follows: In section [I] we review (super-)renormalizable gravity and we show how the Starobinsky model can arise under a suitable ansatz for the form factor. In section III we examine the inflation realization in such a scenario and we provide the corresponding values of observables. Finally, in section IV] we discuss on the obtained results, while section $\nabla$ is devoted to the conclusions.

In what follows we use the signature $(+-\ldots-)$, and the curvature tensor is defined as $R_{\beta \gamma \delta}^{\alpha}=-\partial_{\delta} \Gamma_{\beta \gamma}^{\alpha}+\ldots$, the Ricci tensor as $R_{\mu \nu}=R_{\mu \nu \alpha}^{\alpha}$, and the curvature scalar as $R=g^{\mu \nu} R_{\mu \nu}$, where $g_{\mu \nu}$ is the metric tensor [23].

\section{II. (SUPER-)RENORMALIZABLE GRAVITY}

Let us briefly review super-renormalizable gravity (SRG). In order to be more transparent, we first describe the general theory and then we provide the Starobinsky theory as a special case.

\section{A. General theory}

Super-renormalizable gravity (SRG) is well defined perturbatively at the quantum level. Additionally, at the classical level, the gravitational potential [2], the black hole solutions [1, 3, 4, 24, 25] and the cosmological solutions are singularity-free [2, 26]. The corresponding gravitational Lagrangian is a "non-polynomial" extension of the renormalizable quadratic Stelle theory [27] and it has the following general structure:

$$
\mathcal{L}=R-R_{\mu \nu} \gamma\left(\square_{\Lambda}\right) R^{\mu \nu}+\frac{1}{2} R \gamma\left(\square_{\Lambda}\right) R,
$$

where the "form factor" $\gamma\left(\square_{\Lambda}\right)$ is an "entire function" of the covariant D'Alembertian operator and $\square_{\Lambda}=$ $\square / \Lambda^{2}$, with $\Lambda$ is an invariant mass scale. We mention that non-locality only involves positive powers of the D'Alembertian operator since the two form factors are entire functions. The above theory is not unique, but all the freedom present in the action can be embedded in the function $\gamma\left(\square_{\Lambda}\right)$ [28, 29]. Such function must be interpreted in analogy with the interaction of a photon with a nucleon, that is the form factor for gravity $\gamma\left(\square_{\Lambda}\right)$ could be eventually measured experimentally.

It proves convenient to express the form factor $\gamma\left(\square_{\Lambda}\right)$ introducing a new form factor $V\left(\square_{\Lambda}\right)$ that appears in both the spin two and spin zero part of the propagator, namely

$$
\gamma\left(\square_{\Lambda}\right) \equiv \frac{V\left(\square_{\Lambda}\right)^{-1}-1}{\square}
$$

The above choice is essential in order to have a unitarity ghost-free theory [1 -4], and the classical Lagrangian simplifies to

$$
\mathcal{L}=R-G^{\mu \nu}\left(\frac{V\left(\square_{\Lambda}\right)^{-1}-1}{\square}\right) R_{\mu \nu},
$$

where $G^{\mu \nu}$ is the Einstein's tensor. In order to better clarify this point we recall here the gauge invariant twopoint function for the theory (4):

$$
\left[\mathcal{O}^{-1}(k)\right]_{\text {gauge inv. }}=\frac{V\left(k^{2} / \Lambda^{2}\right)}{k^{2}}\left(P^{(2)}-\frac{P^{(0)}}{2}\right),
$$

where $\mathcal{O}(k)$ is the kinetic operator arising from an expansion of the gravitational action around the flat metric $\eta_{\mu \nu}$ in powers of the graviton field $h_{\mu \nu}$, defined by $g_{\mu \nu}=\eta_{\mu \nu}+h_{\mu \nu}$. Since $V\left(\square_{\Lambda}\right)$ in (3) is a non-polynomial trascendental entire function, only a massless graviton propagates and thus the theory is ghost free. Moreover, the above theory is super-renormalizable [1, 3, 44], as well as unitary and microcausal [28].

In this paper we will mainly focus on the following very natural form factor $V\left(\square_{\Lambda}\right)=e^{-\square_{\Lambda}}$. This ansatz corresponds to a specific and notable super-renormalizable theory, since in 3] it was shown that in this case the graviton propagator is the same with the one obtained starting from a theory of gravity endowed with $\theta$-Poincaré quantum groups of symmetry. Other possible interpretations of such choice come from string filed theory [30 34], stochastic fluctuations of the spacetime at short distance 35] or if we intrinsically assume fractal properties of the spacetime when it is probed at high energy [36, 37, 39 41]. Finally, it would be intriguing to consider the role of the cut-off in defining a minimal length, following the perspective of possible phenomenological explorations (see for instance Ref. [42]).

\section{B. Embedding the Starobinsky $R+\epsilon R^{2}$ model in super-renormalizable gravity}

At the classical level we can truncate the theory (4) at will. In this subsection we focus on the first correction to the Einstein Hilbert action. Since we are dealing with a $D=4$ Friedmann-Lemaître-Robertson-Walker (FLRW) metric, the following term turns out to be topological

$$
\int d^{4} x \sqrt{|g|}\left[3 R_{\mu \nu} R^{\mu \nu}-R^{2}\right]=\text { topological }
$$

which reduces the truncated theory to

$$
\mathcal{L}=R+\frac{1}{6 \Lambda^{2}} R^{2}+O\left(\frac{R \square R}{\Lambda^{4}}\right) .
$$

When $R \square R / \Lambda^{2} \ll R^{2}$ the above Lagrangian reduces to the Starobinsky inflationary model $R+\epsilon R^{2}$, that is the super-renormalizable gravity offers an alternative explanation from its origin at the fundamental level.

In order to obtain a realistic cosmological application of the above model, one needs to include the matter 
Lagrangian $\mathcal{L}_{M}$, corresponding to an energy-momentum tensor $T_{\mu \nu}$. Then the total action in a universe governed by the above truncated super-renormalizable gravity writes as

$$
S=\frac{1}{2 \kappa^{2}} \int d^{4} x \sqrt{|g|}\left[R+\frac{1}{6 \Lambda^{2}} R^{2}+\mathcal{L}_{M}\right],
$$

where $\kappa^{2}=8 \pi G_{N}$ and $G_{N}$ is the gravitational constant.

We conclude that the action (4) can be truncated and then recast in the language of $f(R)$ theories, provided that $f(R)=R+R^{2} /\left(6 \Lambda^{2}\right)$, which is the famous Starobinsky model [16, 19]. The resulting truncated equations of motion are recovered to be

$$
\begin{aligned}
& R_{\mu \nu}-\frac{1}{2} g_{\mu \nu} R+\frac{R}{3 \Lambda^{2}}\left(R_{\mu \nu}-\frac{1}{4} g_{\mu \nu} R\right) \\
& -\frac{1}{3 \Lambda^{2}}\left(g_{\mu \nu} \square R-\nabla_{\mu} \nabla_{\nu} R\right)=8 \pi G_{N} T_{\mu \nu} .
\end{aligned}
$$

It is interesting to mention that starting from an infinite number of higher-derivative terms and under the above suitable truncation, super-renormalizable gravity gives rise to a specific $f(R)$ gravity model, which in some sense is a unification of the different classes in general modified-gravity, i.e. the $f(R)$ and the explicitly higherderivative one.

\section{Embedding the Starobinsky $R+\epsilon R^{2}$ model in renormalizable gravity}

For completeness, in this subsection, we explore another specific choice of the form factor $V(z)$, which introduces the construction of renormalizable gravity $(\mathrm{RG})$. In particular, choosing

$$
V(z)^{-1}=e^{H(z)}
$$

with $z:=-\square_{\Lambda}$ and

$$
H(z)=\frac{1}{2}\left[\gamma_{E}+\Gamma\left(0, z^{2}\right)\right]+\log |z|, \quad \operatorname{Re}\left(z^{2}\right)>0,
$$

the ultraviolet limit of the form factor is

$$
\lim _{z \rightarrow+\infty} V(z)^{-1}=-z \text {. }
$$

Since the entire function $\gamma(z)$ approaches a constant for $|z| \rightarrow+\infty$, this theory embodies the quadratic Stelle action in the ultraviolet limit, but without any ghost pole in the propagator. The form factor cross-connects the quadratic action in the infrared, with an equivalent theory in the ultraviolet. The amplitudes are divergent at each order in the loop expansion and the maximal superficial degree of divergence is four, similarly to the local Stelle's theory. Thus, the theory ceases to be superrenormalizable, but it preserves renormalizability and unitarity as it can be inferred from the general structure of the propagator (5) within the form factor (10).
Let us consider now the intermedium regime. We can write the entire function $H(z)$ as a series

$$
H(z)=\sum_{n=1}^{+\infty}(-1)^{n-1} \frac{z^{2 n}}{2 n n !}=\frac{z^{2}}{2}-\frac{z^{4}}{8}+O\left(z^{6}\right)
$$

The theory for an FLRW spacetime coincides with the Starobinsky theory in the high energy regime, and it is well approximated by the same theory at lower energies too. In particular, the ultraviolet Lagrangian is exactly

$$
\mathcal{L}_{\mathrm{UV}} \approx R+\frac{1}{6 \Lambda^{2}} R^{2}
$$

Applying the Starobinsky and/or Mijic [16, 19] analysis yields a general class of solutions enjoying initial quaside Sitter inflationary behavior. Nevertheless, we are still confronted with the issue of connecting the inflationary epoch to the current FLRW universe phase of expansion. Relation (14) is indeed valid at high curvatures and, as long as $R$ decreases during expansion, higher derivative corrections to (14) may be important, at least for a short period, and may force the exact solutions to be different. Thus, we cannot easily conclude that the theory (12) gives a suitable cosmological expansion, since we should investigate carefully the behavior of the solution in the intermedium regime (13), the reheating and the connection with FLRW universe. Therefore, even though one expects that higher derivative terms are negligible, a deeper analysis of the cosmological solutions in the intermedium regime is needed in order to exclude classical instabilities. We leave such a detailed investigation for a future work.

\section{Differences with string theory}

We finish this section by referring to some differences with the string theory scenarios. In particular, in superstring theory the effective action of the point-particle limit of the ten-dimensional Lagrangian is made out of the usual Einstein-Hilbert term plus the operators

$$
R_{\mu \nu \lambda \rho} R^{\mu \nu \lambda \rho}+a R_{\mu \nu} R^{\mu \nu}+b R^{2},
$$

where $a$ and $b$ are constants. After compactification the four dimensional theory reads as

$$
\mathcal{L}_{\text {string }}=R+\left(\frac{a+1}{3}+b\right) \frac{G_{N} \mathrm{~V}_{6}}{\langle\phi\rangle} R^{2},
$$

where $\mathrm{V}_{6}$ is the compactified volume of the six "extra" dimensions and $\langle\phi\rangle$ is the expectation value of the scalar dilaton field. Therefore, using arguments and taking inspiration from string theory, we might think of fixing uniquely the coefficient in front of $R^{2}$ in the SRG and RG. However, in string theory the preferred values for $a, b$ are $a=-4$ and $b=1$ [19, 43], and thus comparison of (15) and (11) would suggest that $\epsilon=0$, namely no $R^{2}$ 
term is favored by superstring theory. However, these values for the parameter $a$ and $b$ are ambiguous because sensible to a metric field redefinition [4]. On the other hand, in (super-)renormalizable gravity, if a Starobinsky limit of the theory exists, the corresponding coefficient is uniquely fixed. In addition, in such a theory any form factor of the form $V\left(\square_{\Lambda}\right)^{-1} \approx 1+\square_{\Lambda}+O\left(\square_{\Lambda}^{2}\right)$ is compatible with the Starobinsky inflationary model. Thus, we can see that Starobinsky scenario can arise in SRG and RG more effectively than in string theory.

\section{INFLATION IN SUPER-RENORMALIZABLE GRAVITY}

In the previous section we saw that the superrenormalizable theory, when $R \square R / \Lambda^{2} \ll R^{2}$, reduces to the Starobinsky inflationary model $R+\epsilon R^{2}$ with $\epsilon=1 / 6 \Lambda^{2}$. Thus, provided that such an approximation starts to be valid at the beginning of inflation, and hence lasts up to the present epoch, in the proposed SRG model one can obtain the same inflationary picture as in the Starobinsky theory. In the following we summarize the main properties of SRG model introduced in section IIB as they are deduced by $R+\epsilon R^{2}$ Starobinsky model.

In [16] it has been shown that the Starobinsky theory admits an unstable de Sitter phase, and in [19] the authors have shown that the same theory entails a general class of solutions describing with a certain accuracy the cosmological evolution of the Universe. Indeed, such solutions behave initially as a quasi-de Sitter universe, with a slowly decreasing Hubble parameter

$$
H(t) \simeq H_{0}-\frac{\Lambda^{2}}{6}\left(t-t_{0}\right),
$$

with $H_{0}$ the Hubble parameter at the beginning of inflation, namely at time $t_{0}$. Having lasted for a time $t_{\text {osc }}-t_{0} \simeq 6 H_{0} / \Lambda^{2}$, inflation ends and the Universe enters a phase of oscillations with

$$
H(t) \simeq f(t) \cos ^{2}(\omega t)
$$

where $\omega \simeq \Lambda / 2$ and with

$$
f(t) \simeq 1 /\left[3 / \omega+3\left(t-t_{o s c}\right)\right]
$$

representing the damping factor for the amplitude's oscillations. During the oscillatory phase the Universe is reheated and standard-model particles are produced with a reheating temperature [19]

$$
T_{r} \simeq 3 \times 10^{-2} \epsilon^{-\frac{1}{2}} \simeq 4 \times 10^{17} \mathrm{GeV} \sqrt{6 \Lambda^{2} / M_{p}^{2}},
$$

where $M_{p}$ denotes the Planck mass.

It should be noticed that one needs $T_{r} \simeq 10^{10}-10^{16}$ $\mathrm{GeV}$ in order to avoid the monopole problem and properly account for baryogenesis. Finally, after reheating, the Universe enters the usual FLRW phase and then evolves as in the standard cosmological picture ${ }^{1}$.

The $R+\epsilon R^{2}$ scenario entails a spectral index $n_{s}-1 \simeq$ $-2 / N_{e} \simeq-0.04 \times\left(50 / N_{e}\right)$ and a tensor-to-scalar ratio $r \simeq 12 / N_{e}^{2} \simeq 0.005 \times\left(50 / N_{e}\right)^{2}$, parameterized in terms of the e-foldings number $N_{e}$ which is $\Lambda$-independent within a good approximation [22]. Agreement with WMAP nine-year data [17], accounting for $n_{s}=0.971 \pm 0.010$ and $r<0.13$ at $95 \%$ confidence level, is recovered provided that $N_{e} \simeq 50-55$ [17, 22]. Moreover the amplitude of initial perturbations requires [22],

$$
\frac{\Lambda}{M_{p}} \simeq 1.5 \times 10^{-5} \times\left(50 / N_{e}\right) \text {. }
$$

Taking into account the constraints from cosmological perturbations in (20), the reheating temperature turns out to be $T_{r} \simeq 5 \times 10^{12} \mathrm{GeV}$. Assuming supersymmetry, a potential issue related to such a value of $T_{r}$ is the overproduction of gravitinos, which would take place at reheating temperatures $T_{r} \geq 10^{10} \mathrm{GeV}$ for a wide range of gravitino's masses [45, 46]. The role of a $R^{3}$ term in lowering the reheating temperature down to $T_{r} \simeq 10^{9}$ $\mathrm{GeV}$ has been addressed in [22]. Such a term also originates within the framework of the higher-dimensional extension of the SRG theories we have addressed in this paper. We leave to a forthcoming paper ${ }^{2}$ the task of establishing a quantitative relation with the study developed in [22].

In light of these considerations, the SRG model constructed in subsection $\llbracket$ A provides a suitable inflationary scenario which mimics very well the Starobinsky theory, to which it reduces at low curvatures $R \square R / \Lambda^{2} \ll$ $R^{2}$. Additionally, the SRG model can be related to FLRW universe at late times. However, as we discussed in subsection $\mathrm{IC}$, this is not the case for the RG model introduced there, since even though the latter model reduces asymptotically to $R+\epsilon R^{2}$ gravity for $R \rightarrow \infty$, it may deviate from the Starobinsky theory as long as the curvature $R$ decreases, and thus the relation between the RG model and the FLRW universe will be non-trivial in this case. Finally, we remark that the mass scale $\Lambda$, which represents a cutoff for the higher derivative terms, is well below the Planck scale, and thus it may have observational consequences for highly energetic but still subPlanckian phenomena.

\section{DISCUSSION}

Let us make some remarks concerning the equivalence of our particular SRG model of Lagrangian (7) with the

\footnotetext{
1 A cosmological constant is still needed in order to describe the late-time acceleration.

2 We acknowledge the referee for pointing out to us the issue of gravitinos over-production.
} 
$f(R)=R+\epsilon R^{2}$ gravity. As we analyzed in subsection IIB the Starobinsky model can be obtained through a coherent truncation of SRG, and thus in section III we used this result in order to describe the inflationary scenario in SRG. However, some significant difference could arise between SRG and Starobinsky model.

As it is well known, a general property of $f(R)$ gravity is the appearance of an extra scalar curvaton degree of freedom. Such a scalar degree of freedom can be explicitly expressed performing a Weyl transformation of the metric tensor $\tilde{g}_{\mu \nu}(x) \equiv \exp [\sqrt{2 / 3} k \phi] g_{\mu \nu}(x)$, which maps the original $f(R)$ theory of the Jordan frame into General Relativity plus a scalar field in the Einstein frame, provided that the scalar field potential is $V(\phi)=$ $\left[f^{\prime}(R) \tilde{R}-f(R)\right] / 2 k^{2} f^{\prime}(R)^{2}$, where $f^{\prime}(R) \equiv \partial f(R) / \partial R$ and $\tilde{R}$ is the Ricci scalar constructed with the $\tilde{g}_{\mu \nu}$ metric tensor (for instance see [4]]). Therefore, the phenomenology of the $f(R)$ scenario could be obtained in terms of the scalar field $\phi$ in the Einstein frame. For example the number of e-folds during inflation is recovered to be $N_{e}=\int_{t}^{t_{f}} H d t \simeq \int_{\phi_{f}}^{\phi} V / V^{\prime} M_{p}^{2} d \phi$, where $t_{f}$ denotes the time at the end of inflation.

What is more important in the above picture is that the extra scalar degree of freedom is responsible for scalar perturbations during inflation. Thus, in the Einstein frame the slow-roll inflation parameters are obtained in terms of the scalar field $\phi$ as $\epsilon_{\phi}=\left(M_{p}^{2} / 2\right)\left(V^{\prime} / V\right)^{2}$ and $\eta_{\phi}=M_{p}^{2} V^{\prime \prime} / V$ [48], which lead to the slope of the power spectrum: $n_{s}=1+2 \eta_{\phi}-6 \epsilon_{\phi}$. Finally, the amplitude of initial perturbations is given by $\Delta_{R}^{2}=$ $M_{p}^{4} V /\left(24 \pi^{2} \epsilon_{\phi}\right)$, which is observationally estimated [48] to be $\left(V(\phi) / \epsilon_{\phi}\right)^{\frac{1}{4}} \simeq 6.6 \times 10^{16} \mathrm{GeV}$.

Therefore, as we analyzed in detail in the previous section, in the specific case of $f(R)=R+\epsilon R^{2}$ gravity the above analysis allows to estimate the $\Lambda$ parameter as $\Lambda / M_{p} \sim 10^{-5}$ [22, 49], and correspondingly for the power spectrum $n_{s}=1+2 \eta-6 \epsilon \simeq 1-2 / N_{e}$, for the tensor primordial spectrum $n_{t} \simeq-2 \epsilon \simeq-3 / 2 N_{e}^{2}$, and for the tensor-to-scalar ratio $r=16 \epsilon \simeq 12 / N_{e}^{2}$ [49]. In other words, in the $f(R)=R+\epsilon R^{2}$ model the induced extra scalar degree of freedom $\phi$ is responsible for the generation of primordial scalar perturbations which in turn are used to estimate the $\epsilon$ parameter, or the $\Lambda$ parameter in the SRG case.

However, a general feature of SRG and RG which makes a fundamental difference with $R+\epsilon R^{2}$ theory, is the fact that no extra scalar degree of freedom seems to exist in such theories. In fact, in [1-4] it has been shown that, after quantization of the gravitational field on flat Minkowskian background metric, only the usual massless spin-two gravitons and no other extra degrees of freedom are found. We stress that this result has been demonstrated only on a flat background and it is not trivially extended to a curved spacetime. However, if confirmed, this would in part invalidate the analysis performed in subsection III In particular, at a classical background level the evolutionary picture described in subsection III is safely valid, since the truncation introduced in subsection $\amalg \mathrm{B}$ is valid as long as $R \square R / \Lambda^{2} \ll R^{2}$. However, at perturbation level the absence of the extra degree of freedom (if confirmed) could leave the scenario without a mechanism to generate primordial perturbations during the inflationary epoch, and thus one should need to find other such mechanisms, for instance introducing by hand additional scalar or alternatively vector fields [50] (such a formulation would also imply that the obtained bound on the parameter $\Lambda \sim 10^{-5} \times M_{p}$ would need to change).

Nevertheless, note that SRG can have an advantage comparing to $f(R)=R+\epsilon R^{2}$ gravity, concerning the generation of non-gaussianities. In particular, it is known that the effective scalar degree of freedom of $f(R)=$ $R+\epsilon R^{2}$ gravity is not capable to correctly produce the non-gaussianities. On the other hand, SRG has still the choice to add higher order terms in the Lagrangian (7), which could lead to non-gaussian perturbations. Since upper bounds on non-gausianities may be soon imposed through the experimental investigation of CMBR by the WMAP [51] and the Planck satellites, it will furnish an immediate opportunity to falsify models with large nongaussianities, in order to distinguish among the plethora of inflationary models of the literature. We mention that the amount and shape of deviations from a Gaussian distribution of primordial density reveals a critical dependence on the details of the given inflationary model [52, 53], and thus it could be a crucial test for the SRG origin of Starobinsky scenario.

\section{CONCLUSIONS}

In this work we investigated the realization of inflation in the context of (super-)renormalizable gravity, which is a gravitational theory constructed consistently with the basic and minimal requirements of a quantum gravity, being well defined perturbatively at the quantum level. The corresponding gravitational Lagrangian is a "nonlocal" extension of the renormalizable quadratic Stelle theory, expressed using a form factor consisting of an entire function. Thus, at the classical level we can truncate the theory in order to obtain many subclasses, one of them being the Starobinsky inflationary model. Similarly, imposing suitably a different choice for the form factor, instead of super-renormalizability we can obtain a renormalizable theory that can also give rise to Starobinsky model, though not so efficiently. In summary, (super)renormalizable gravity offers an explanation of the origin of Starobinsky model at the fundamental level.

The approximate realization of the Starobinsky model in (super-)renormalizable gravity allows us to use the inflationary results of that model in order to examine inflation in (super-)renormalizable gravity. In particular, we find that the solutions of the theory behave initially as a quasi-de Sitter universe, with a slowly decreasing Hubble parameter, and thus inflation can end and the Universe 
enter a phase of damping oscillations that reheats it.

However, there is still a difference between Starobinsky -type inflation in $f(R)$ gravity and the Starobinsky-type inflation in (super-)renormalizable gravity. In particular, while in the former there appears an extra scalar curvaton degree of freedom which is responsible for generating the perturbations during inflation, in the latter case no extra scalar degree of freedom seems to exist. Although this has not be proven for general spacetimes but only for flat ones, if confirmed it would make necessary an incorporation of a new mechanism to generate the observationally required primordial perturbations. However, inflation in (super-)renormalizable gravity has an advantage, namely that it could lead to non-gaussian perturbations, which is not the case in Starobinsky-type $f(R)$ gravity inflation. Such a prospect would act as an additional asset for (super-)renormalizable gravity.

\section{Acknowledgments}

We warmly thank S. Alexander and S. Odintsov for valuable discussions and comments to the paper. F. Briscese is a Marie Curie fellow of the Istituto Nazionale di Alta Matematica Francesco Severi. A. Marcianò gratefully acknowledges support by Perimeter Institute during the early stage of this work and financial support through Career grant NSF (part of this work has been carried out while at Princeton University). Research at Perimeter Institute is supported by the Government of Canada through Industry Canada and by the Province of Ontario through the Ministry of Research \& Innovation. The research of ENS is implemented within the framework of the Action "Supporting Postdoctoral Researchers" of the Operational Program "Education and Lifelong Learning" (Action's Beneficiary: General Secretariat for Research and Technology), and is co-financed by the European Social Fund (ESF) and the Greek State.
[1] L. Modesto, Phys. Rev. D 86, 044005 (2012).

[2] T. Biswas, E. Gerwick, T. Koivisto and A. Mazumdar, Phys. Rev. Lett. 108, 031101 (2012).

[3] L. Modesto arXiv:1202.3151 [hep-th]].

[4] S. Alexander, A. Marciano, L. Modesto, Phys. Rev. D 85 (2012)124030.

[5] L. Modesto arXiv:1202.0008 [hep-th]]; L. Modesto arXiv:1206.2648 [hep-th]].

[6] J. W. Moffat, Phys. Rev. D 41, 1177 (1990); B. J. Hand and J. W. Moffat, Phys. Rev. D 43, 1896 (1991); D. Evens, J. W. Moffat, G. Kleppe and R. P. Woodard, Phys. Rev. D 43, 499 (1991); N. J. Cornish, Mod. Phys. Lett. A 7, 1895 (1992); N. J. Cornish, Int. J. Mod. Phys. A 7, 6121 (1992); N. J. Cornish, Mod. Phys. Lett. A 7, 631 (1992); J. W. Moffat, Eur. Phys. J. Plus 126, 43 (2011).

[7] S. 'i. Nojiri and S. D. Odintsov, eConf C 0602061, 06 (2006) [Int. J. Geom. Meth. Mod. Phys. 4, 115 (2007)].

[8] A. De Felice and S. Tsujikawa, Living Rev. Rel. 13, 3 (2010).

[9] T. Clifton, P. G. Ferreira, A. Padilla and C. Skordis, Phys. Rept. 513, 1 (2012).

[10] S. 'i. Nojiri and S. D. Odintsov, Phys. Lett. B 659, 821 (2008).

[11] S. Capozziello, E. Elizalde, S. 'i. Nojiri and S. D. Odintsov, Phys. Lett. B 671, 193 (2009).

[12] S. 'i. Nojiri and S. D. Odintsov, Phys. Rept. 505, 59 (2011).

[13] P. Hořava, Phys. Rev. D 79, 084008 (2009); A. E. Gumrukcuoglu, S. Mukohyama, and A. Wang, Phys. Rev. D 85, 064042 (2012); P. Hořava and C. M. Melby-Thompson, Phys. Rev. D 82, 064027 (2010); C. Bogdanos and E. N. Saridakis, Class. Quant. Grav. 27, 075005 (2010); Y. -F. Cai and E. N. Saridakis,
JCAP 0910, 020 (2009); F. Briscese, Y. Rodriguez and G. A. Gonzalez, Found. Phys. 42 (2012).

[14] S. Alexander, J. Magueijo and A. Marciano, Phys. Rev. D 86, 064025 (2012).

[15] S. Deser and R. P. Woodard, Phys. Rev. Lett. 99 (2007) 111301.

[16] A. A. Starobinsky, Phys. Lett. B 91, 99 (1980).

[17] G. Hinshaw, D. Larson, E. Komatsu, D. N. Spergel, C. L. Bennett, J. Dunkley, M. R. Nolta and M. Halpern et al., arXiv:1212.5226 [astro-ph.CO].

[18] D. Bailin, A. Love, D. Y. Wong. Phys.Lett. B 165 (1985) 270.

[19] M. B. Mijić, M. S. Morris, Wai-Mo Suen, Phys. Rev. D34, 2934-2946 (1986).

[20] E. V. Arbuzova, A. D. Dolgov, L. Reverberi, JCAP 02 (2012) 049.

[21] S. 'i. Nojiri and S. D. Odintsov, Phys. Lett. B 576, 5 (2003).

[22] S. V. Ketov and A. A. Starobinsky, Phys. Rev. D 83, 063512 (2011).

[23] A. A. Starobinsky, JETP Lett. 86, 157 (2007).

[24] L. Modesto, J. W. Moffat and P. Nicolini, Phys. Lett. B 695, 397 (2011).

[25] P. Nicolini, A. Smailagic and E. Spallucci, arXiv:hep-th/0507226 P. Nicolini, J. Phys. A 38, L631 (2005); P. Nicolini, A. Smailagic and E. Spallucci, Phys. Lett. B 632, 547 (2006); P. Nicolini, arXiv:1202.2102 [hep-th]; P. Nicolini, Int. J. Mod. Phys. A 24 (2009) 1229.

[26] N. Barnaby, T. Biswas, J. M. Cline, JHEP 0704056 (2007); T. Biswas, A. Mazumdar, W. Siegel, JCAP 0603 (2006) 009; S. Alexander, T. Biswas, Phys. Rev. D 80 (2009) 023501 Phys. Rev. D 80 023501; T. Biswas, T. Koivisto, A. Mazumdar, JCAP 1011 (2010) 008; T. 
Biswas, A. S. Koshelev, A. Mazumdar, S. Yu. Vernov, JCAP 1208 (2012) 024.

[27] K. S. Stelle, Phys. Rev. D 16, 953 (1977).

[28] G. V. Efimov, Nonlocal Interactions [in Russian], Nauka, Moskow (1977); N. V. Krasnikov, Teoreticheskaya $i$ Matematicheskaya Fizika, Vol. 73, No. 2, 235-244, November 1986.

[29] E. T. Tomboulis, hep-th/9702146.

[30] P. -M. Ho, Class. Quant. Grav. 21, 2641 (2004).

[31] V. A. Kostelecky and S. Samuel, Phys. Rev. D 42, 1289 (1990).

[32] T. Biswas, J. Kapusta and A. Reddy, arXiv:1201.1580 [hep-th].

[33] E. Witten, Nucl. Phys. B 268, 253 (1986).

[34] L. Brekke, P. G. O. Freund, M. Olson and E. Witten, Nucl. Phys. B 302, 365 (1988).

[35] K. Namsrai, Nonlocal Quantum Field Theory and Stochastic Quantum Mechanics, D. Reidel Publishing Company, Dordrecht, Holland (1986).

[36] G. Calcagni, JHEP 1003 (2010) 120.

[37] G. Calcagni, Phys. Rev. Lett. 104 (2010) 251301.

[38] G. Calcagni and G. Nardelli, arXiv:1210.2754 [hep-th].

[39] G. Calcagni, JHEP 1201 (2012) 065.

[40] G. Calcagni, Phys. Lett. B 697, 251 (2011).

[41] L. Modesto and P. Nicolini, Phys. Rev. D 81 (2010) 104040.

[42] M. Sprenger, M. Bleicher and P. Nicolini, Class. Quant. Grav. 28 (2011) 235019.

[43] M. Maggiore, Nucl. Phys. B 525 (1998) 413.
[44] D. J. Gross and E. Witten, Nucl. Phys. B 277, 1 (1986).

[45] M. Kawasaki and T. Moroi, Prog. Theor. Phys. 93, 879 (1995) hep-ph/9403364 hep-ph/9403061.

[46] T. Moroi, hep-ph/9503210.

[47] K. Bamba, S. Capozziello, S. 'i. Nojiri and S. D. Odintsov, Astrophys. Space Sci. 342, 155 (2012).

[48] A. R. Liddle and D. H. Lyth, Cosmological Inflation and Large-scale Structure, Cambridge University Press, Cambridge (2000).

[49] S. Kaneda, S. V. Ketov and N. Watanabe, Mod. Phys. Lett. A 25, 2753 (2010).

[50] K. Dimopoulos, M. Karciauskas and J. M. Wagstaff, Phys. Rev. D 81, 023522 (2010); D. V. Gal'tsov and E. A. Davydov, Proc. Steklov Inst. Math. 272, 119 (2011); A. Maleknejad and M. M. Sheikh-Jabbari, arXiv:1102.1513 [hep-ph]; S. Alexander, A. Marciano, D. Spergel, arXiv:1107.0318 [hep-th]; D.V. Gal'tsov, E.A. Davydov, arXiv:1112.2943 [hep-th]; E. Elizalde, A.J. Lopez-Revelles, S.D. Odintsov, S.Yu. Vernov, arXiv:1201.4302 [hep-th]; P. Adshead and M. Wyman, Phys. Rev. Lett. 108, 261302 (2012); M. M. Anber and L. Sorbo, Phys. Rev. D 85, 123537 (2012).

[51] P. Creminelli, A. Nicolis, L. Senatore, M. Tegmark and M. Zaldarriaga, JCAP 0605, 004 (2006).

[52] N. Bartolo, E. Komatsu, S. Matarrese and A. Riotto, Phys. Rept. 402, 103 (2004).

[53] X. Chen, Adv. Astron. 2010, 638979 (2010). 